\title{
Oxidative Stress in Cyclosporine-Induced Hypertension: Evidence of Beneficial Effects or Tolerance Development With Nitrate Therapy
}

\author{
F. Reis, P. Rocha-Pereira, E. Teixeira de Lemos, B. Parada, S. Baptista, A. Figueiredo, A. Santos-Silva, \\ C. Costa-Almeida, A. Mota, and F. Teixeira
}

\begin{abstract}
The aim of this study was to evaluate the effect of cyclosporine (CsA) on oxidative stress as well as the use of a nitric oxide (NO) donor, the organic nitrate isosorbide-5-mononitrate (Is-5-Mn), to prevent or reverse CsA-induced toxicity, namely on the vascular NO-cGMP pathway or on oxidative equilibrium. The following rat groups $(n=8)$ were tested: (1) a control group; (2) the CsA group (5 mg/kg/d for 7 weeks); (3) the Is-5-Mn group (150 mg/kg/d, twice a day for 7 weeks); (4) the preventive group (Is-5-Mn + CsA) treated for 2 weeks with Is-5-Mn only, and thereafter with both drugs for 7 weeks; (5) the curative group (CsA + Is-5-Mn) beginning 7 weeks after CsA, and following thereafter with both drugs for 5 weeks. The following parameters were evaluated: aortic cNOS activity and cGMP content; plasma levels of lipid peroxidation (malondialdehyde [MDA] levels); antioxidant capacity (glutathione peroxidase [GPx] and superoxide dismutase [SOD] activities, total antioxidant status, and vitamins $\mathrm{A}, \mathrm{C}$, and $\mathrm{E}$ ); and peroxynitrite formation (3-nitrotyrosine [3-NT] content). Is-5-Mn + CsA therapy showed, when compared with the CsA group, total prevention of CsAinduced NO and cGMP attenuation, and no relevant influence on antioxidant indices, as well as on MDA and 3-NT levels. However, when compared with this CsA group, the curative group (CsA + Is-5-Mn) showed NO-cGMP values only partially reversed, and an enhancement in lipid peroxidation $(5.6 \pm 1.4 \mathrm{vs} 12.78 \pm 3.63 \mu \mathrm{mol} / \mathrm{L} ; P<.05)$ and in peroxynitrite formation ( $16.7 \%$ incidence of positives vs $83.3 \%$ incidence of positives). Our data suggested that nitrate therapy may provide a valid choice to prevent CsA-induced NO-cGMP decrease, without a negative influence on the oxidative equilibrium. However, when the local environment is adverse, as occurs after CsA therapy, Is-5-Mn seemed to enhance the CsA-induced oxidative stress, promoting even worse deleterious effects, probably through the generation of the cytotoxic ROS peroxynitrite.
\end{abstract}

$\mathrm{P}^{\mathrm{o}}$ OSTTRANSPLANTATION HYPERTENSION (HT) is one of the major cyclosporine (CsA)-induced side effects. Because this HT effect may produce serious cardiovascular risks that jeopardize a long-lasting, successful organ transplant, anti-HT therapy is advised. ${ }^{1}$ As long as the main cause for HT remains to be fully explained, the choice of the most appropriate anti-HT drug must consider its efficiency to control blood pressure (BP) values for a specific type of transplant, as well as the hypothetical side effects and potential interference with immunosuppressive ability. ${ }^{2}$ Actually, all classic anti-HT drugs have showed benefits and disadvantages. ${ }^{3,4}$

Increased vascular resistance associated with CsA-induced HT has been attributed to impaired vascular reactivity, in
From the Institute of Pharmacology and Experimental Therapeutics (F.R., E.T.L, B.P., S.B., F.T.) and Institute of Physiology (C.C.-A.), Faculty of Medicine, Coimbra University, Coimbra, Portugal; Chemistry Department (P.R.-P.), Beira Interior University, Covilhã, Portugal; Department of Urology and Renal Transplantation (B.P., A.F., A.M.), Coimbra University Hospital, Coimbra, Portugal; Biochemistry Service (A.S.-S.), Faculty of Pharmacy, Porto University, Porto, Portugal; Molecular and Cellular Biology Institute (P.R.-P., A.S.-S.), Porto University, Porto, Portugal.

Address reprint requests to Prof Frederico Teixeira, Therapeutics Unit, Institute of Pharmacology and Experimental Therapeutics, Faculty of Medicine, Coimbra University, 3004-504 Coimbra, Portugal. E-mail: fredjt@ci.uc.pt

(c) 2007 by Elsevier Inc. All rights reserved. 360 Park Avenue South, New York, NY 10010-1710 
which the attenuation of vasodilatation might play a crucial role. ${ }^{5,6}$ Previous studies from our group, as well as from others, have shown a disequilibrium in the nitric oxide (NO)/cyclic guanosine-3',5'-monophosphate (cGMP) system, which may contribute to the vascular hyperreactivity. ${ }^{7,8}$ The NO system is essential to the relaxation of vascular smooth muscles cells (VSMC) and plays a pathophysiological role in reactive oxygen or nitrogen species (ROS) generation. ${ }^{9}$ In addition, oxidative stress could also have a key role in CsA-associated cardiovascular toxicity. ${ }^{10,11}$ Thus, NO-donor supplementation might have, in theory, a positive influence by preventing or normalizing NO-cGMP pathway dysfunction; it may also influence oxidative equilibrium and ROS generation.

This animal model study sought to evaluate the effects of CsA on vascular NO, cGMP content, and oxidative equilibrium, by evaluating both antioxidant and ROS formation markers. Moreover, we intended to estimate the preventive or the reversal influence of an NO donor, the organic nitrate isosorbide-5-mononitrate (Is-5-Mn), on vasomotor and oxidative stress effects of CsA.

\section{METHODS}

Male Wistar rats (280-300 g; Charles River Laboratories Inc, Barcelona, Spain) were maintained in an air conditioned room, subjected to 12-hour dark/light cycles, given standard laboratory rat chow (IPM-R20, Letica, Barcelona, Spain), and allowed free access to tap water. Animal experiments were conducted according to the European Communities Council Directives on Animal Care. The rats were divided into 5 groups (each with 8 rats) treating each, by oral administration, with the following: control group-only orange juice for 7 weeks; CsA group $-5 \mathrm{mg} / \mathrm{kg}$ body weight (BW)/d CsA (Sandimmune Neoral, Novartis Farma, Lisbon, Portugal), dissolved in orange juice, for 7 weeks; Is-5-Mn group $-150 \mathrm{mg} / \mathrm{kg} \mathrm{BW} / \mathrm{d}$, twice a day, Is-5-Mn (Monopront, Ferraz-Lynce SA, Lisbon, Portugal) for 7 weeks; preventive Is-5-Mn group (Is-5-Mn + CsA) -150 $\mathrm{mg} / \mathrm{kg} / \mathrm{d}$, twice a day, Is-5-Mn for 2 weeks and the same dose of Is-5-Mn + CsA (5 mg/kg/d) for an additional 7 weeks; curative/ regressive Is-5-Mn group (CsA + Is-5-Mn) $-5 \mathrm{mg} / \mathrm{kg} / \mathrm{d}$ CsA for 7 weeks and the same dose of CsA $+150 \mathrm{mg} / \mathrm{kg} / \mathrm{d}$, twice a day, Is-5-Mn for an additional 5 weeks. All administrations were performed through an appropriate esophageal cannula at about the same hour of the day (17:00 hours) for control and CsA-treated rats. In order to have a daily nitrate-low/free period of about 4 to 5 hours to overcome hypothetical organic nitrate tolerance, Is-5-Mn twice a day administration was made asymmetrically at 10:00 and 17:00 hours. All determinations were performed at the end of the scheduled treatments.

At the end of the treatments the rats were subjected to intraperitoneal anesthesia with $2 \mathrm{mg} / \mathrm{kg}$ BW of a $2: 1$ (v:v) $50 \mathrm{mg} / \mathrm{mL}$ ketamine (Ketalar, Parke-Davis, Laboratórios Pfeizer, Lda, Seixal, Portugal) solution in $2.5 \%$ chlorpromazine (Largactil, RhônePoulenc Rorer, Laboratórios Vitória, Amadora, Portugal), and then the animals were sacrificed by cervical dislocation. Blood was withdrawn from the jugular vein by venipuncture, collected into tubes with or without anticoagulant, and centrifuged to obtain serum and plasma samples ( $160 \mathrm{~g}$ for 10 minutes at $20^{\circ} \mathrm{C}$ ). To perform vascular tissue studies, the aorta was immediately removed and placed in ice-cold Krebs' buffer, carefully cleaned of adherent fat and connective tissue, and cut into segments, with special care to preserve the integrity of the endothelial layer.

The aortic cNOS activity was assessed through assay of the conversion of $\left[{ }^{3} \mathrm{H}\right]$-L-arginine into $\left[{ }^{3} \mathrm{H}\right]$-L-citrulline. ${ }^{12}$ The aortic segments were homogenized in a solution containing Tris- $\mathrm{HCl}(250$ $\mathrm{mmol} / \mathrm{L})$, EDTA (10 mmol/L), and EGTA (10 mmol/L), at $\mathrm{pH} 7.4$, and centrifuged thereafter $\left(18,500 \mathrm{~g}, 5\right.$ minutes at $\left.4^{\circ} \mathrm{C}\right)$. Supernates were recovered to evaluate protein concentration using bovine serum albumin (BSA) as the standard, ${ }^{13}$ and to further assess cNOS activity. The extracts were incubated at $37^{\circ} \mathrm{C}$ for 60 minutes in a buffer ( $\mathrm{pH} 7.4)$ containing Tris- $\mathrm{HCl}(50 \mathrm{mmol} / \mathrm{L})$, tetrahydrobiopterin (BH4: $6 \mu \mathrm{mol} / \mathrm{L}$ ), flavin adenine dinucleotide (FAD: 2 $\mu \mathrm{mol} / \mathrm{L}$ ), nicotinamide adenine dinucleotide phosphate (NADPH: $1 \mathrm{mmol} / \mathrm{L}$ ), and $10 \mu \mathrm{Ci} / \mathrm{mL}$ of $\left[{ }^{3} \mathrm{H}\right]-\mathrm{L}$-arginine (Amersham Pharmacia Biotech, Buckinghamshire, UK). Constitutive NOS activity was measured by incubating the medium in $\mathrm{Ca}^{2+}(1 \mathrm{mmol} / \mathrm{L})$ and by discounting the blank value of a sample incubated with the inhibitors of both isoforms: L-N $\omega$-nitro-arginine (L-NNA: 1 $\mathrm{mmol} / \mathrm{L}$ ) + aminoguanidine (AMG: $1 \mathrm{mmol} / \mathrm{L}$ ). The reaction was stopped by adding $1.5 \mathrm{~mL}$ of Dowex $50 \mathrm{~W}-\mathrm{X} 8$ resin (forma $\mathrm{Na}^{+}$; Bio-Rad, Richmond, Calif, USA) and $5 \mathrm{~mL}$ of distilled water. The amount of $\left[{ }^{3} \mathrm{H}\right]-\mathrm{L}$-citrulline in the sample supernates was then quantified with liquid scintillation (ICN, Biomedicals Inc, Irvine, Calif, USA) in a spectrophotometer (Packard Tri-Carb 2000CA, Downers Grove, Ill, USA). cNOS activity was expressed as pmol of $\left[{ }^{3} \mathrm{H}\right]$-L-citrulline/mg protein/min.

The protocols for aortic cGMP extraction and quantification were performed as previously described, ${ }^{7}$ using a commercially available immunoassay kit (R\&D Systems, Minneapolis, Minn, USA). The assay was performed in the presence of the phosphodiesterase inhibitor 3-isobutyl-1-methylxanthine (IBMX: $2 \mathrm{mmol} / \mathrm{L}$ ), in order to prevent spontaneous nucleotide degradation. As a positive control test for cGMP quantification, we used sodium nitroprusside $(0.1 \mathrm{mmol} / \mathrm{L})$. All assays were performed in duplicate. Values were expressed as $\mathrm{pmol} / \mathrm{mg}$ protein, which was determined by the Bradford method, ${ }^{13}$ using BSA as a standard.

Superoxide dismutase (SOD) and glutathione peroxidase (GPx) activities, expressed in $\mathrm{IU} / \mathrm{g} \mathrm{Hb}$, were assessed using commercially available kits (Ransod and Ransel, respectively, from Randox Lab Ltd, Crumlin, UK). Total antioxidant status (TAS) was evaluated in serum samples by using commercially available kits (Total Antioxidant Status, Randox Lab Ltd), ${ }^{14}$ with results expressed as $\mathrm{mmol} / \mathrm{L}$.

Whole blood, collected into tubes containing $\mathrm{K}_{3}$-EDTA as an anticoagulant, was used to evaluate vitamin $\mathrm{C}$ concentrations. The samples were treated with a previous extraction protocol with 0.3 $\mathrm{mol} / \mathrm{L}$ of trichloroacetic acid (TCA) for each milliliter of whole blood. After a continuous vortex agitation for 20 minutes in a dark environment, the samples were centrifuged at $1000 \mathrm{~g}$ for 10 minutes to extract the organic phase. Sodium acetate $4.5 \mathrm{~mol} / \mathrm{L}(\mathrm{pH} 6.2)$ was then added to the supernate and incubated under agitation for 2 minutes at $37^{\circ} \mathrm{C}$ in an ascorbate-oxidase strip, which was thereafter placed in a solution of ortho-phenylene-diamine (OPD $0.1 \%$ in physiological saline solution). The sample was finally homogenized and incubated at $37^{\circ} \mathrm{C}$ for 30 minutes under continuous vortex agitation. Vitamin $\mathrm{C}$ concentration was assessed by high performance liquid chromatography (HPLC) with fluorescence detection (Shimadzu RF-10A XL) under the following conditions: room temperature, excitation $\lambda=355 \mathrm{~nm}$ and emission $\lambda=435$ $\mathrm{nm}$, flux of $1 \mathrm{~mL} / \mathrm{min}$, injection volume of $20 \mu \mathrm{L}$ and 8 minutes running time. The concentrations were obtained using a standard of vitamin $\mathrm{C}$ with results expressed in $\mathrm{mg} / \mathrm{dL}$. 
Serum vitamin A and E levels were measured by HPLC using a commercially available kit (Vitamin A/E by HPLC, Bio-Rad Lab $\mathrm{GmbH}$, Munich, Germany) according to the protocol previously described. ${ }^{15}$ Prior to analysis, the samples were vortexed for 1 minute with an internal standard of $\alpha$-tocopherol; ammonium sulfate was added to differentiate the 2 phases by centrifugation (10,000g for 3 minutes). The upper phase was recovered for quantification of vitamins $\mathrm{A}$ and $\mathrm{E}$ by HPLC in an isocratic chromatographic system with UV detection (HP 1100 detector, Bio-Rad) with the following conditions: $45^{\circ} \mathrm{C}$ temperature, $\lambda=340$ $\mathrm{nm}$ for vitamin A detection followed by $\lambda=295 \mathrm{~nm}$ for vitamin E, flux of $2.5 \mathrm{~mL} / \mathrm{min}$, injection volume of $20 \mu \mathrm{L}$ and running time of 7.5 minutes. The values were obtained by comparison with the internal standard and expressed in $\mathrm{g} / \mathrm{dL}$ and $\mu \mathrm{g} / \mathrm{mL}$ for vitamins $\mathrm{A}$ and $\mathrm{E}$, respectively.

Products of lipid peroxidation, namely, malondialdehyde (MDA), were evaluated by the thiobarbituric acid (TBA) assay. ${ }^{16}$ The assay mixture consisted of $0.1 \mathrm{~mL}$ serum, $0.4 \mathrm{~mL} 0.9 \%$ $\mathrm{NaCl}, 0.5 \mathrm{~mL} 3 \%$ sodium dodecylsulfate (SDS), and $3 \mathrm{~mL}$ TBA reagent (containing equal parts of $0.8 \%$ aqueous TBA and acetic acid); the mixture was heated for 75 minutes at $95^{\circ} \mathrm{C}$ and, thereafter, $1 \mathrm{~mL}$ cold $0.9 \% \mathrm{NaCl}$ and extracted with $5 \mathrm{~mL}$ $n$-butanol. After centrifugation at $730 \mathrm{~g}$ for 15 minutes at $4^{\circ} \mathrm{C}$, the organic phase was analyzed spectrophotometrically at $532 \mathrm{~nm}$, using 1,1,3,3-tetramethoxypropane as an external standard. The results were expressed as $\mu \mathrm{mol} / \mathrm{L}$ of MDA.

Plasma 3-nitrotyrosine (3-NT) concentration, which is an index of peroxynitrite formation, was quantified using an enzymatic immunoassay (HyCult Biotechnology b.v., Uden, The Netherlands) with results expressed in $\mathrm{nmol} / \mathrm{L}$.

Results are presented as mean values \pm standard errors (SEM). Differences between groups were tested by performing ANOVA followed by the Fisher Protected Least Significant Difference (PLSD) test, using Statview 4.53 software from Abacus Concepts Inc. (Berkeley, Calif, USA). Differences were considered significant at $P<.05$. The monotherapy groups of CsA and Is-5-Mn were compared with the controls and the Is-5-Mn + CsA; the CsA + Is-5-Mn groups were compared with the CsA alone.

\section{RESULTS}

The CsA group showed aortic cNOS activity lower than controls $(1.6 \pm 0.2$ vs $3.0 \pm 0.2 ; P<.05$; Fig 1$)$; the Is- 5 -Mn group showed similar values $(2.9 \pm 0.3)$ to those of the control group; the preventive group (Is-5-Mn + CsA), also similar values to those of the latter groups, but significantly higher values than those of the CsA group $(3.2 \pm 0.3 ; P<$ $.05)$, suggesting a full prevention of CsA-induced cNOS activity reduction (Fig 1A). However, the curative group (CsA + Is-5-Mn) showed only a trend toward reversion of the CsA effect $(2.2 \pm 0.2$; Fig $1 \mathrm{~A})$.

In the CsA group there was a decreased arterial cGMP content compared with the control group $(9.6 \pm 2.2$ vs $22.0 \pm$ $3.5 ; P<.05$; Fig 1B). This effect was totally prevented and reversed by Is-5-Mn used as a preventive (Is-5-Mn + CsA group: $36.1 \pm 3.9 ; P<.001)$ or curative (CsA + Is-5-Mn group: $23.1 \pm 3.4 ; P<.05$ ) therapy (Fig 1B). However, as occurred with the Is-5-Mn group, and in opposition to the CsA + Is-5-Mn group, the effect of Is-5-Mn when used preventively with CsA was an increased cGMP content even above the values achieved in the control group (Fig 1B).
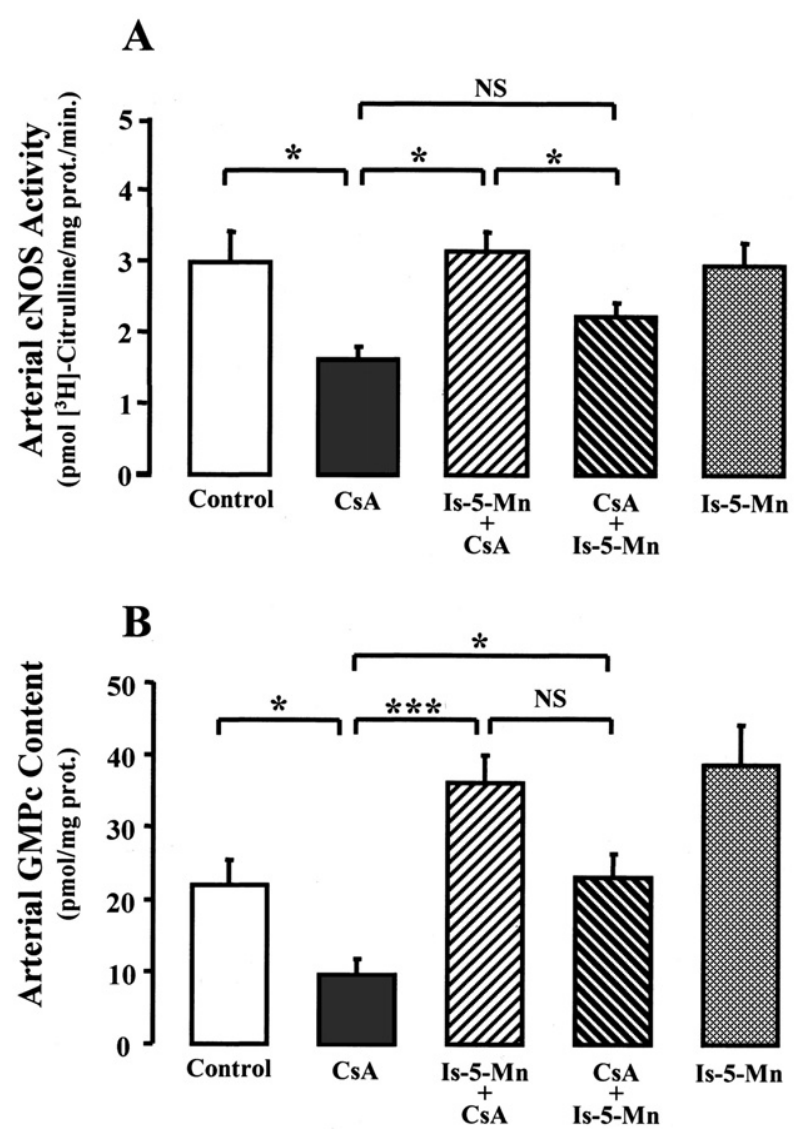

Fig 1. Arterial cNOS activity (A) and cGMP content (B) in the 5 rat groups under study at the end of the treatments. Data are expressed as means \pm SEM of 8 separate values (rats). Significant differences between the groups are expressed: ${ }^{\star} P<.05$ and ${ }^{* \star *} P<.001$; NS: non-significant.

SOD and GPx activity were similar in all groups (Table $1)$, except the CsA group, which displayed increased GPx activity $(P<.05)$. Accordingly, the TAS index value was also similar in all groups (Table 1). Concerning blood vitamin $\mathrm{C}$ concentrations, only 1 significant difference was found, namely, a significantly lower value in the CsA + Is-5-Mn group $(P<.05)$ compared with the CsA group. All other groups showed similar TAS values compared with the control group (Table 1). The concentrations of serum vitamins $\mathrm{A}$ and $\mathrm{E}$, however, showed a marked increase in the CsA group compared with the control $(P<.001)$. This effect was prevented by nitrate when used as preventive therapy (Is-5-Mn + CsA: $P<.001$ ), but it was not reversed by nitrate when used as curative therapy (CsA + Is-5-Mn; Table 1).

Serum MDA concentration, an indicator of lipid peroxidation, showed a trend to higher values in the CsA group $(5.6 \pm 2.4)$ compared with the controls $(1.8 \pm 0.6$; Fig $2 \mathrm{~A})$. While in the preventive group $(5.7 \pm 0.6)$, the MDA concentration was not significantly different from that of the CsA group, the MDA in the curative group was even worse 
Table 1. Parameters of Antioxidant Status

\begin{tabular}{lccccc}
\hline & Control & CsA & Is-5-Mn & Is-5-Mn + CsA & CsA + Is-5-Mn \\
\hline SOD activity (U/g Hg) & $1486 \pm 75$ & $1483 \pm 53$ & $1334 \pm 66$ & $1352 \pm 65$ & $1417 \pm 61$ \\
GPx activity (U/g Hg) & $107 \pm 3$ & $122 \pm 2^{*}$ & $104 \pm 4$ & $118 \pm 3$ & $111 \pm 5$ \\
TAS (mmol/L) & $0.77 \pm 0.02$ & $0.79 \pm 0.02$ & $0.79 \pm 0.02$ & $0.72 \pm 0.01$ & $0.78 \pm 0.02$ \\
Vit C (mg/dL) & $0.35 \pm 0.02$ & $0.38 \pm 0.05$ & $0.35 \pm 0.02$ & $0.37 \pm 0.03$ & $0.28 \pm 0.02^{\dagger}$ \\
Vit A ( $\mu \mathrm{g} / \mathrm{dL})$ & $42.26 \pm 1.18$ & $120.05 \pm 5.48^{\star \star \star}$ & $38.38 \pm 2.73$ & $40.34 \pm 1.79^{\dagger+\dagger}$ & $102.68 \pm 26.45$ \\
Vit E (mg/L) & $0.43 \pm 0.07$ & $1.27 \pm 0.20^{\star \star \star}$ & $0.36 \pm 0.11$ & $0.38 \pm 0.13^{\dagger+\dagger}$ & $0.98 \pm 0.14$ \\
\hline
\end{tabular}

Data are expressed as means \pm SEM of 8 separate values (rats). Significant differences between the groups are expressed: ${ }^{\star} P<.05$ and ${ }^{\star \star \star} P<.001$ vs the control group; ${ }^{\dagger} P<.05$ and ${ }^{\dagger+} P<.001$ vs the CsA group.

$(12.8 \pm 3.6 ; P<.05)$, showing a significant increase in lipid peroxidation compared with all other groups (Fig 2A). The MDA/TAS index, which is considered an index of oxidative balance, showed identical variations, demonstrating a notorious increase in oxidative stress among the curative group compared with the CsA group $(P<.05)$, as well as with all other groups (Fig 2B).

The values of plasma 3-NT, an indicator of peroxynitrite formation, were analyzed in terms of presence of levels above the detection limit of $0.023 \mathrm{nmol} / \mathrm{L}$ (incidence of positives). As shown in Fig 2C, all values from the controls were undetectable ( $0 \%$ incidence of positives). In the CsA group, only 1 of the 6 values showed a higher 3 -NT content ( $2.5 \mathrm{nmol} / \mathrm{L})$, representing $16.7 \%$ of positives (Fig 2C). A similar pattern (1 in 6) was obtained in the Is-5-Mn + CsA group, showing no attenuation or aggravation of the CsA effects, being the positive value analyzed, of $0.9 \mathrm{nmol} / \mathrm{L}$. However, among the CsA + Is-5-Mn group, 5 of 6 values were positive, representing an incidence of $83.3 \%$, which was marked by different from the other groups (Fig 2C).

\section{DISCUSSION}

One possibility to explain CsA-induced vascular dysfunction associated with HT development is impaired NO-cGMP pathway, ${ }^{6-8}$ but the results described in the literature during the last years are diverse and contradictory. ${ }^{17,18}$ Another possibility is that CsA promotes the development of oxidative stress as the cause for the cardiovascular toxicity. ${ }^{10,11}$ Our previous data using a rat model of CsA-induced HT indicated the existence of a vascular NO-cGMP impairment, which could not be merely attributed to a lower L-arginine utilization, because NOS substrate could not completely prevent CsAinduced $\mathrm{HT}^{19}$ in opposition to the positive influence of Is-5-Mn administration as a preventive for the CsA-induced $\mathrm{BP}$ rise. ${ }^{20}$ However, the beneficial effect of Is-5-Mn when tested as a preventive therapy was not achieved when Is-5-Mn was supplemented in a curative fashion. ${ }^{21}$ Thus, we have considered highly relevant the evaluation of the influence on oxidative equilibrium of 2 types of Is-5-Mn treatments. One cannot underestimate the close relationship between $\mathrm{NO}$ and reactive oxygen species (ROS) formation, ${ }^{9}$ as well as the possibility of tolerance development for the class of organic nitrates, ${ }^{22,23}$ including Is-5-Mn.

The present study confirmed that CsA attenuates the NO-cGMP system, as previously reported by us and by other groups., ${ }^{7,8,24}$ The reduction in aortic cNOS activity might be better explained by an effect on its complex enzymatic profile rather than by a reduction of L-arginine content or consumption, since previous studies have demonstrated that L-arginine supplementation was unable to prevent CsA-induced HT. ${ }^{19,25}$ Thus, an effect on the enzymatic cofactors of cNOS may not be excluded, as already documented in other pathophysiological conditions. ${ }^{26} \mathrm{Un}$ der certain dysfunctional circumstances, such as cofactor deficiency, NOS activity may produce superoxide anion instead of NO, a state described as "uncoupled NOS function."27,28 This possibility for the CsA effect would explain the reduced $\mathrm{NO}$ and cGMP content as well as the associated cardiovascular toxicity. Moreover, it would also explain the lack of efficacy of L-arginine supplementation and suggest a potential beneficial effect of a different type of NO complement, such as the organic nitrate Is-5-Mn, which was our choice for this study. In agreement with this hypothesis, the present study showed the inhibition (or trend to reversion) of CsA-induced decrease in arterial NOS activity and cGMP content, using a preventive or a curative Is-5-Mn treatment, respectively. Considering the NO donor properties of Is-5-Mn, the increased cGMP content obtained may be viewed as an expected result, a direct corollary of increased cellular NO levels after intracellular conversion. Nevertheless, it should be emphasized that the cGMP levels achieved in the CsA + Is-5-Mn group, which were comparable with the values in the control group, were not higher than those of the other Is-5-Mn groups, raising the possibility that the NO yielded by nitrate in this group (CsA + Is-5-Mn) may be used to form other components rather than cGMP. Considering the ability of Is-5-Mn to prevent CsAinduced HT (but not to reverse the BP rise), as previously reported by us, ${ }^{2}$ there exists the possibility of oxidative stress development by the curative Is-5-Mn treatment due to the formation of reactive oxygen and nitrogen species.

We intended to evaluate the effect of the treatments in both antioxidant and pro-oxidant indices. The results of SOD and GPx activities, as well as of TAS, indicated that CsA had no relevant influence on the antioxidant condition. No differences were also observed for vitamin $\mathrm{C}$ values. However, there was a surprising effect of CsA on vitamin A and $\mathrm{E}$ levels, which were higher than control values. These data apparently contradict the increased lipid peroxidation reported by others and also evident in our results. The 

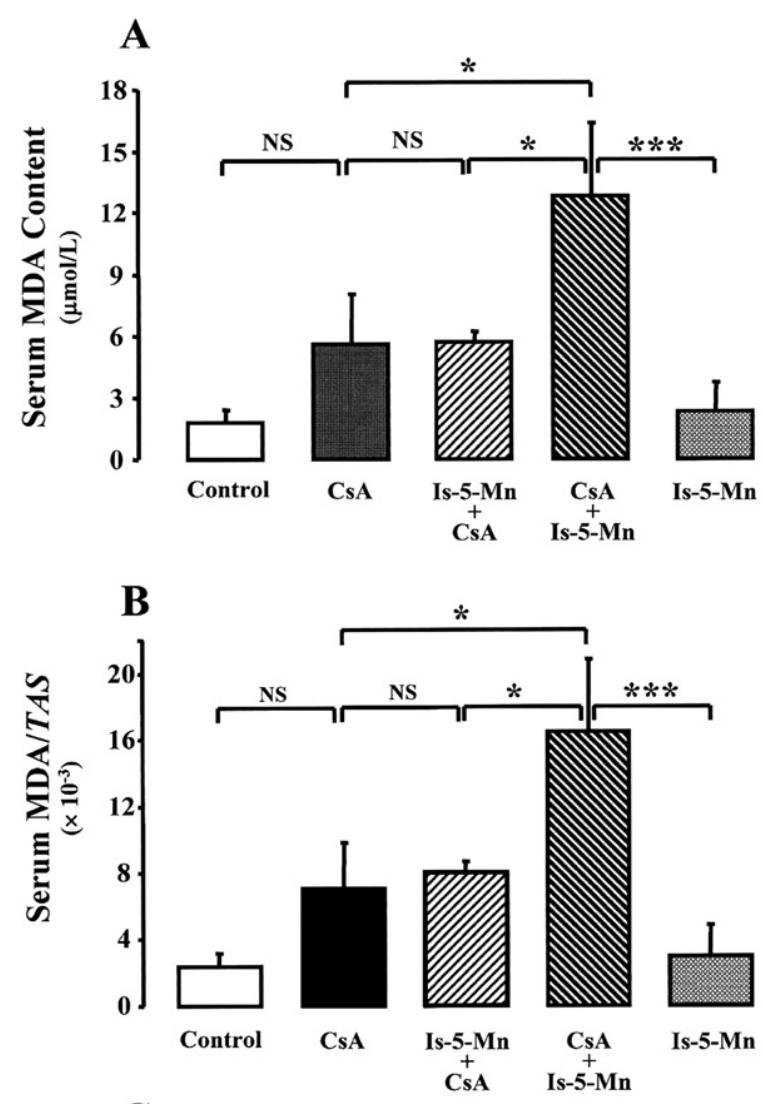

C

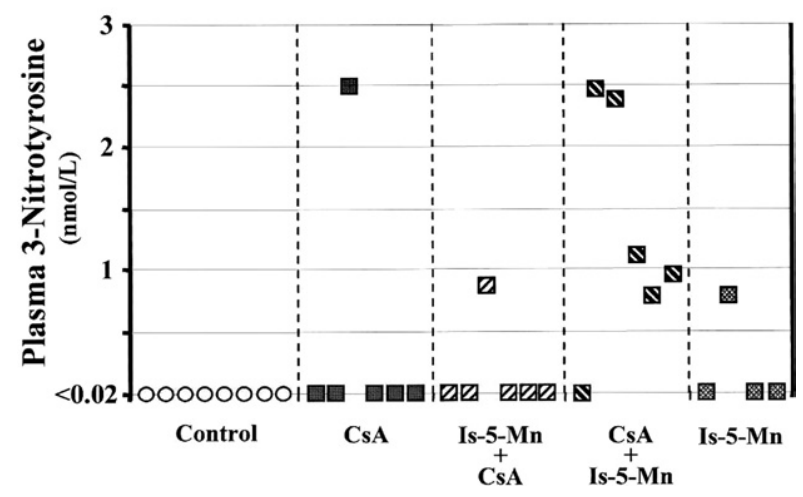

Fig 2. Serum MDA content (A), MDATTAS index (B), and plasma nitrotyrosine levels (C) in the 5 rat groups under study at the end of the treatments. Data are expressed as means \pm SEM of $n$ separate values (rats). Significant differences between the groups are expressed: ${ }^{\star} P<.05$ and ${ }^{\star \star \star} P<.001$; NS: non-significant.

increased vitamin $\mathrm{A}$ and $\mathrm{E}$ concentrations may result from a compensatory mechanism to face oxidative stress. Also surprising was the observation of a similar result in the CsA + Is-5-Mn group, which was the group unable to reverse the CsA-induced BP rise, ${ }^{21}$ and the one that showed several aggravated cardiovascular parameters. An explanation for the rise in those vitamins is the mobilization (in the 2 rat groups in which the oxidative aggression was more evident) of the exogenous vitamin A and E content, supplied by diet (Panlab, SL, containing 15,000 UI/ $/ \mathrm{kg}$ of vitamin A and $30 \mathrm{mg} / \mathrm{kg}$ of alpha-tocopherol), into the circulation in an attempt to compensate for development of oxidative stress. Moreover, considering the lipid soluble nature of these vitamins, the rise could also result in part from associated hyperlipidemia. ${ }^{21}$

Our data agree with other studies which also showed a rise in vitamin $\mathrm{E}$ associated with CsA treatment, namely among transplanted patients. ${ }^{29}$ Actually, other authors have also attributed the rise in antioxidant activity to a possible compensatory effect. ${ }^{30}$ Interestingly, no important modifications were observed in other antioxidant parameters, suggesting that the CsA-induced cardiovascular toxicity might be better explained by ROS formation associated with NO-cGMP attenuation rather than a failure of the antioxidant defense systems. Moreover, another interesting result was the aggravation of CsA-induced MDA formation in the CsA + Is-5-Mn group, but not in the nitrate group used as preventive therapy (Is-5-Mn + CsA). These data favor oxidative stress accentuation when the nitrate is given after the establishment of CsA-induced modifications. This hypothesis was further corroborated by the MDA/TAS index and especially by the striking rise in 3-NT formation (an index of peroxynitrite production) in the curative CsA + Is-5-Mn group. This finding also confirmed our previous hypothesis of an accentuated ROS formation when nitrate is supplied after CsA treatment.

Thus, we may now raise a consistent explanation for the CsA effects on the NO-cGMP pathway as well as for the distinct effects of preventive and curative nitrate therapy on parameters of vasomotor and oxidative equilibrium. It seems evident that CsA may promote an "uncoupling NO synthase state," previously reported in the literature for other cardiovascular pathophysiological situations. ${ }^{27,28}$ This state not only reduced NO and cGMP content, as shown by our results, but also increased superoxide anion formation, thus consistently explaining the CsA effects on vascular reactivity and cardiovascular toxicity. Concerning the Is-5-Mn effects when given as preventive and curative therapy, it seems probable that previous Is-5-Mn treatment prevents NO and cGMP reduction, thus avoiding the side effects of CsA. However, when given after CsA, the NO supplied by the nitrate reacts with the superoxide anion hypothetically originated by CsA action, thus originating the formation of peroxynitrite and explaining not only the lower recuperation of cGMP content in the CsA + Is-5-Mn group, as opposed to the other Is-5-Mn groups, but also even the increased toxicity when compared with the CsA group. The higher 3-NT incidence in this CsA + Is-5-Mn group, as well as the notable increase in MDA content, confirmed the increased peroxynitrite formation as well as the augmented lipid peroxidation. Peroxynitrite, therefore, as one of the main deleterious NO-derived ROS, produces further injurious effects on the cardiovascular system, namely on several distinct vascular and platelet mechanisms, including lipid peroxidation, augmentation of platelet aggregation or modification of LDL and promotion of its pro-atherogenic interaction with macrophages. ${ }^{31,32}$ 
The increased ROS and oxidative stress in the curative nitrate treatment might be viewed as one form of nitrate tolerance. Actually, in the preventive group we observed beneficial effects on BP and on other cardiovascular parameters negatively affected by CsA, and no significant interference with oxidative equilibrium and ROS formation. In the curative group, the lack of a positive influence or even an aggravation on the CsA-induced HT as well as on other cardiovascular indices, was associated with increased ROS formation and lipid peroxidation. We should emphasize that one of the main possibilities for the long-time investigated mechanisms of nitrate tolerance is the possibility of ROS formation, namely through vascular superoxide anion generation which will conjugate with the NO supplied by the nitrate, as previously suggested for the class of organic nitrates. ${ }^{33,34}$

Thus, our study suggested that nitrate therapy should be considered a valid choice for prevention of CsA-induced cardiovascular modifications because, in conjugation with the beneficial effects already demonstrated, no negative interference on the oxidative equilibrium and ROS formation was demonstrated. However, this study also strongly indicated that Is-5-Mn therapy after the changes originated by CsA were already established is a nonrecommended choice because not only was the HT not reversed, but also ROS formation was promoted, in a mechanism that could be viewed as nitrate tolerance.

\section{ACKNOWLEDGMENTS}

This study had the kind collaboration of Novartis Farma (Lisbon, Portugal) which supplied the cyclosporine (Sandimmune Neoral) and of Ferraz-Lynce (Lisbon, Portugal) which supplied the isosorbide-5mononitrate (Monopront).

\section{REFERENCES}

1. Taler SJ, Textor SC, Canzanello VJ, et al: Cyclosporininduced hypertension: incidence, pathogenesis and management. Drug Saf 20:437, 1999

2. Olyaei AJ, de Mattos AM, Bennett WM: A practical guide to the management of hypertension in renal transplant recipients. Drugs 58:1011, 1999

3. Martinez-Castelao A, Hueso M, Sanz V, et al: Treatment of hypertension after renal transplantation: long-term efficacy of verapamil, enalapril, and doxazosin. Kidney Int Suppl 68:S130, 1998

4. del Castillo D, Campistol JM, Guirado L, et al: Efficacy and safety of losartan in the treatment of hypertension in renal transplant recipients. Kidney Int Suppl 68:S135, 1998

5. Cartier R, Dagenais F, Hollmann C, et al: Chronic exposure to cyclosporine affects endothelial and smooth muscle reactivity in the rat aorta. Ann Thorac Surg 58:789, 1994

6. Rego A, Vargas R, Wroblewska B, et al: Attenuation of vascular relaxation and cyclic GMP responses by cyclosporin A. J Pharmacol Exp Ther 252:165, 1990

7. Santiago M, Reis F, Almeida L, et al: Impairment of vascular and platelet nitric oxide and $3^{\prime}, 5^{\prime}$ cyclic guanosine monophosphate content in cyclosporin A-induced hypertensive rats. Fund Clin Pharmacol 17:43, 2003

8. Oriji GK, Keiser HR: Role of nitric oxide in cyclosporin A-induced hypertension. Hypertension 32:849, 1998

9. Beckman J: The ABC's of the reactions between nitric oxide, superoxide, peroxynitrite and superoxide dismutase. Oxygen 99Sunrise Free Radical School 1, 1999
10. Beutler TM, Cottet-Maire F, Krauskopf A, et al: Does cyclosporin A generate free radicals? TiPS 21:288, 2000

11. Nishiyama A, Kobori H, Fukui T, et al: Role of angiotensin II and reactive oxygen species in cyclosporine A-dependent hypertension. Hypertension 42:754, 2003

12. Zalba G, Beaumont FJ, San Jose G, et al: Is the balance between nitric oxide and superoxide altered in spontaneously hypertensive rats with endothelial dysfunction? Nephrol Dial Transplant 16(suppl 1):2, 2001

13. Bradford MM: A rapid and sensitive method for quantitation of microgram quantities of protein utilizing the principle of protein-dye binding. Anal Biochem 72:248, 1976

14. Miller NJ, Rice-Evans C, Davies MJ, et al: A novel method for measuring antioxidant capacity and its application to monitoring the antioxidant status in premature neonates. Clin Sci (Lond) 84:407, 1993

15. Bierig JG, Tolliver TJ, Catignani GL: Simultaneous determination of alpha-tocopherol and retinol in plasma or red cells by high pressure liquid chromatography. Ann J Clin Nutr 32:2143, 1979

16. Saldeen T, Li D, Mehta JL: Differential effects of $\alpha$ - and $\gamma$-tocopherol on low-density lipoprotein oxidation, superoxide activity, platelet aggregation and arterial thrombogenesis. J Am Coll Cardiol 34:1208, 1999

17. O'Neil GS, Chester AH, Kushwaha S, et al: Cyclosporin treatment does not impair the release of nitric oxide in human coronary arteries. Br Heart J 66:212, 1991

18. Stroes ES, Luscher TF, Groot FG, et al: Cyclosporin A increases nitric oxide activity in vivo. Hypertension 29:570, 1997

19. Reis F, Santiago M, Almeida L, et al: Isosorbide-5-mononitrate and L-arginine effect on the cyclosporin-induced arterial hypertension and vascular nitric oxide impairment. In Tooke J, Shore A, Whatmore J (eds): The Microcirculation and Vascular Biology. Bologna, Italy: Monduzzi Editore S.p.A.; 2002, p257

20. Reis F, Almeida L, Alcobia T, et al: Isosorbide-5-mononitrate treatment prevents cyclosporin A-induced platelet hyperactivation and the underlying nitric oxide-cyclic guanosine- $3^{\prime}, 5^{\prime}$-monophosphate disturbances. Thromb Res 110:107, 2003

21. Reis F, Ponte L, Rocha L, et al: Curative isosorbide-5-mononitrate treatment, in opposition to the beneficial preventive one, aggravates the prothrombotic and proconstrictor state in cyclosporineinduced hypertensive rats. Clin Exp Pharmacol Physiol 32:640, 2005

22. Horowitz JD: Tolerance induction during therapy with longacting nitrates: how extensive is the "collateral damage"? Cardiovasc Drug Ther 18:11, 2004

23. Csont T, Ferdinandy P: Cardioprotective effects of glyceryl trinitrate: beyond vascular nitrate tolerance. Pharmacol Ther 105:57, 2005

24. Bartholomeusz B, Hardy KJ, Philips PA: Modulation of nitric oxide improves cyclosporin A-induced hypertension in rats and primates. J Hum Hypertens 12:839, 1998

25. Lassila M, Santisteban J, Finckenberg P, et al: Vascular changes in cyclosporine A-induced hypertension and nephrotoxicity in spontaneously hypertensive rats on high-sodium diet. J Physiol Pharmacol 52:21, 2001

26. Katusic ZS: Vascular endothelial dysfunction: does tetrahydrobiopterin play a role? Am J Physiol Heart Circ Physiol 281:H981, 2001

27. Stroes ES, Hijmering M, Zandvoort M, et al: Origin of superoxide production by nitric oxid synthase. FEBS Lett 438:161, 1998

28. Vasquez-Vivar J, Kalyanaraman B, Martasek P, et al: Superoxide generation by endothelial nitric oxid synthase: the influence of cofactors. Proc Natl Acad Sci USA 95:9220, 2000

29. de Lorgeril M, Boissonnat $\mathrm{P}$, Salen $\mathrm{P}$, et al: The beneficial effect of dietary antioxidant supplementation on platelet aggregation and cyclosporine treatment in heart transplant recipients. Transplantation 58:193, 1994

30. Parra-Cid T, Conejo Garcia JR, Carballo Alvarez F, et al: Antioxidant nutrients protect against CyA nephrotoxicity. Toxicology 189:99, 2003 
31. Brown AS, Moro MA, Masse JM, et al: Nitric oxidedependent and independent effects on human platelets treated with peroxynitrite. Cardiovasc Res 40:380, 1998

32. Graham A, Hogg N, Kalyanaraman B, et al: Peroxynitrite modification of low-density lipoprotein leads to recognition by the macrophage scavenger receptor. FEBS Lett 330:181, 1993
33. Watanabe H, Kakihama M, Ohtsuka S, et al: Randomized, double-blind, placebo-controlled study of supplemental vitamin C on attenuation of the development of nitrate tolerance. Circulation 97:886, 1998

34. Schwemmer M, Bassenge E: New approaches to overcome tolerance to nitrates. Cardiovasc Drug Ther 17:159, 2003 